\title{
Idiosyncratic body motion influences person recognition
}

\author{
Karin S. Pilz and lan M. Thornton ${ }^{\mathrm{b}}$

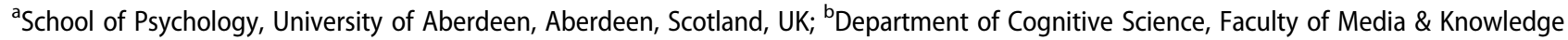 \\ Science, University of Malta, Msida, Malta
}

\begin{abstract}
Person recognition is an important human ability. The main source of information we use to recognize people is the face. However, there is a variety of other information that contributes to person recognition, and the face is almost exclusively perceived in the presence of a moving body. Here, we used recent motion capture and computer animation techniques to quantitatively explore the impact of body motion on person recognition. Participants were familiarized with two animated avatars each performing the same basic sequence of karate actions with slight idiosyncratic differences in the body movements. The body of both avatars was the same, but they differed in their facial identity and body movements. In a subsequent recognition task, participants saw avatars whose facial identity consisted of morphs between the learned individuals. Across trials, each avatar was seen animated with sequences taken from both of the learned movement patterns. Participants were asked to judge the identity of the avatars. The avatars that contained the two original heads were predominantly identified by their facial identity regardless of body motion. More importantly however, participants identified the ambiguous avatar primarily based on its body motion. This clearly shows that body motion can affect the perception of identity. Our results also highlight the importance of taking into account the face in the context of a body rather than solely concentrating on facial information for person recognition.
\end{abstract}

ARTICLE HISTORY

Received 27 June 2016

Accepted 26 August 2016

\section{KEYWORDS}

Person recognition; face recognition; body motion; animation technique
Person recognition is a vitally important, frequently practiced and finely tuned perceptual ability. Visually, the face is probably the main source of information we use when recognizing others, and it is certainly the best studied (Bruce \& Young, 2012). However, although we are very good at recognizing people from their faces alone, outside of the laboratory we are rarely required to do so. Real-world decisions about identity, age, gender, actions, intentions and emotional state also commonly involve the whole body (for review see Johnson \& Shiffrar, 2013; Knoblich, Thornton, Grosjean, \& Shiffrar, 2006). Furthermore, bodies are typically in motion. Our need to interpret and predict the actions of others appears to have led our visual system to become particularly sensitive to human movement (Blake \& Shiffrar, 2007; Giese \& Poggio, 2003; Johansson, 1973; Thompson \& Parasuraman, 2012; Troje, 2002). In the current paper, we use recent motion capture and computer animation techniques to quantitatively explore the impact of body motion on person recognition decisions.
To date, the stimuli that have mainly been used to study the perception of human movement are pointlight figures, which consist of point-lights that are attached to the major joints of an actor (Johansson, 1973). When set in motion, these lights create a convincing image of a moving person in the absence of any other cues. Previous studies have shown that we are very good at recognizing actions, and can also judge the gender, age, emotional state and even identity from body motion alone (Agnew, Phillips, \& Pilz, 2016; Cutting \& Kozlowski, 1977; Dittrich, Troscianko, Lea, \& Morgan, 1996; Johansson, 1973; Kozlowski \& Cutting, 1977; Loula, Prasad, Harber, \& Shiffrar, 2005; Pollick, Kay, Heim, \& Stringer, 2005; Spencer, Sekuler, Bennett, Giese, \& Pilz, 2016; Vanrie \& Verfaillie, 2004).

The very nature of point-light figures, however, limits their use when questions about body form, facial identity or scene context are also of interest. To overcome some limitations of point-light walkers, several studies have used dynamic whole body stimuli to investigate person recognition in connection with individual body motion (Bruce, Henderson,

CONTACT Karin S. Pilz k.s.pilz@abdn.ac.uk @ School of Psychology, University of Aberdeen, Aberdeen, Scotland, UK

๑ 2016 Informa UK Limited, trading as Taylor \& Francis Group 
Newman, \& Burton, 2001; Burton, Wilson, Cowan, \& Bruce, 1999; Hahn, O'Toole, \& Phillips, 2016; Liu, Seetzen, Burton, \& Chaudhuri, 2003; Robbins \& Coltheart, 2015; Simhi \& Yovel, 2016; Yovel \& O'Toole, 2016). In an early paper, Burton et al. (1999) tested participants' ability to identify people captured by a video security system while entering a building. They found that familiar but not unfamiliar individuals were recognized quite successfully based on their body motion. More recently, Simhi and Yovel (2016) conducted a similar study and showed that even for unfamiliar person recognition, body motion during learning can facilitate performance. It has been suggested that body motion plays a particular important role for person recognition when the person is distant and the facial information suboptimal (Hahn et al., 2016; Rice, Phillips, \& O'Toole, 2013; Yovel \& O'Toole, 2016).

Even though the stimuli used in the studies mentioned above are more naturalistic than previously used point-light figures, they often have the disadvantage of providing strong identity cues from individual body structure/shape, as well as from external features, such as clothing and hairstyle. While most studies controlled for the effects of external cues by keeping them constant across conditions, their availability, together with individual differences in body form, makes it difficult to completely disentangle the effect of body motion on person recognition from other prominent cues. A solution to overcome the limitations of point-light walkers and videos when studying person recognition is to use animated avatars.

The development of photo-realistic avatars has progressed tremendously over the last decade (Powell, Corbett, \& Powell, 2016), driven by demands from the movie and games industries (Spanlang et al., 2013), as well as virtual interfaces for learning software (Kao \& Harrell, 2015), or virtual guides in museums as a tool to interact with users and visitors (Gesellensetter, Krämer, \& Wachsmuth, 2005; Panayiotopoulos et al., 2005). For the scientist, avatars are highly appealing as they enable the presentation of high-resolution motion-captured actions together with realistic body information (Thornton, 2006). Rather than removing details of the form, as in point-light stimuli, here the trick is to provide it, but to render it irrelevant or redundant with respect to the hypotheses under investigation. A number of studies have already successfully used animation techniques to explore facial motion using head models (Griesser, Cunningham, Wallraven, \& Bülthoff, 2007; Hill, Jinno, \& Johnston, 2003; Knappmeyer, Thornton, \& Bülthoff, 2003; Wallraven, Breidt, Cunningham, \& Bülthoff, 2008). Regarding the use of avatars to investigate dynamic person recognition, the literature is less extensive. In fact, to our knowledge, there is only one previous study that has used avatars to study the impact of body motion on person recognition (Pilz, Vuong, Bülthoff, \& Thornton, 2011). Pilz et al. (2011) attached individual 3D head models to an avatar that was either moving away or towards the participant, and found better performance for approaching compared to receding stimuli, i.e., the movement of an approaching person alone affected recognition performance.

In the current paper, we take a further step to directly investigate the effect of individual body motion on the encoding and later recognition of identity. We made use of an approach that had previously been used to investigate the impact of facial motion on the recognition of identity: Knappmeyer et al. (2003) familiarized participants with different 3D head models that were animated with naturalistic facial movements motion-captured from human actors. When the learned models were presented with facial movements performed by a different actor during test, recognition performance was impaired.

In the current experiment, participants were first familiarized with two animated avatars each performing the same basic sequence of karate actions with slight idiosyncratic differences in the body movements. The body of both avatars was the same, but they differed in their heads and body movements. Test stimuli were created by morphing the heads of the individually learned avatars in steps of $10 \%$. The heads were then attached to the same body that was used during learning. The resulting 11 avatars were then animated with sequences from the two body motions that had been used during the familiarization phase. Participants had to judge the identity of the avatars. Using such animated whole body avatars we were able to investigate whether individual body motion is encoded together with the facial identity of a person. We expected participants to judge the ambiguous avatar based on the motion sequence it was animated with. 


\section{Methods}

\section{Participants}

Sixteen right-handed participants aged 19 to $26(M=$ 22.2 years, nine males) participated in this study. They were recruited from the MPI subject pool and received 8 Euro/hour. All participants had normal or correctedto-normal vision, were naïve regarding the purpose of the experiment, and gave written informed consent.

\section{Stimuli}

Two heads of the MPI face database (which we here arbitrarily labelled as Joachim and Denis) were morphed in steps of $10 \%$ (Blanz \& Vetter, 1999; Figure 1). The resulting 11 heads were placed on the same body model obtained from aXYZ-design (www. axyz-design.com). It should to be noted that the skin tone of the faces was adjusted to match the skin tone of the avatar. In addition, only internal facial features were used such that hairstyle, ears and the face outline was the same for all stimuli. The resulting avatars were animated with the same karate sequence performed by two different actors-one a professional karate player, the other one an amateur. Stimuli were processed using 3D Studio Max (http://www.autodesk. com). The resulting sequences consisted of 468 frames and were played back at a frame rate of 25 fames/ second (fps), resulting in a total of 18.72 seconds per sequence. The frames extended a visual angle of $11.5^{\circ} \times 17^{\circ}$.

\section{Procedure}

Participants were seated in a sound attenuated room, approximately $60 \mathrm{~cm}$ away from the screen. The experiment consisted of a learning phase and a test phase. During learning, participants watched the two original avatars, that is $100 \%$ Joachim and $100 \%$ Denis. Joachim was animated with the professional karate movements, and Denis was animated with the amateur karate movements. The avatars were alternating on the screen, each being repeated 10 times. To familiarize participants with the two avatars, participants filled out a questionnaire concerning facial features (e.g., please list the most prominent facial features) and character traits (e.g., please list the most prominent character traits), while watching the avatars in motion. We decided to use such questionnaire to enhance learning, as it has been shown that judgments about character traits increase the depth of processing compared to simple sex judgments, for example (Bower \& Karlin, 1974; Pilz, Bülthoff, \& Vuong, 2009). We have successfully used similar learning procedures in previous studies (Knappmeyer et al., 2003; Pilz et al., 2009; Pilz, Thornton, \& Bülthoff, 2006; Pilz, Vuong et al., 2011). We would like to highlight that participants were only

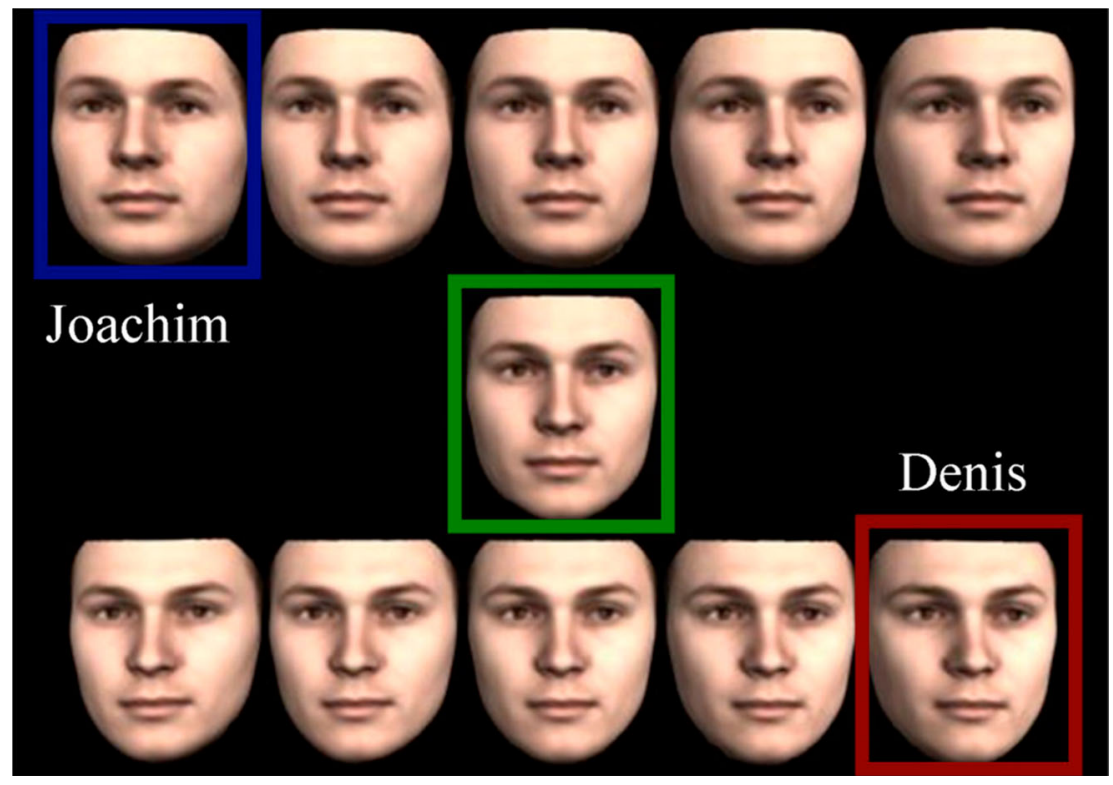

Figure 1. Morph sequence of the two heads. Joachim, upper left, was morphed towards Denis in steps of $10 \%$. The average morph$50 \%$ morph between Denis and Joachim — can be seen in the middle. 

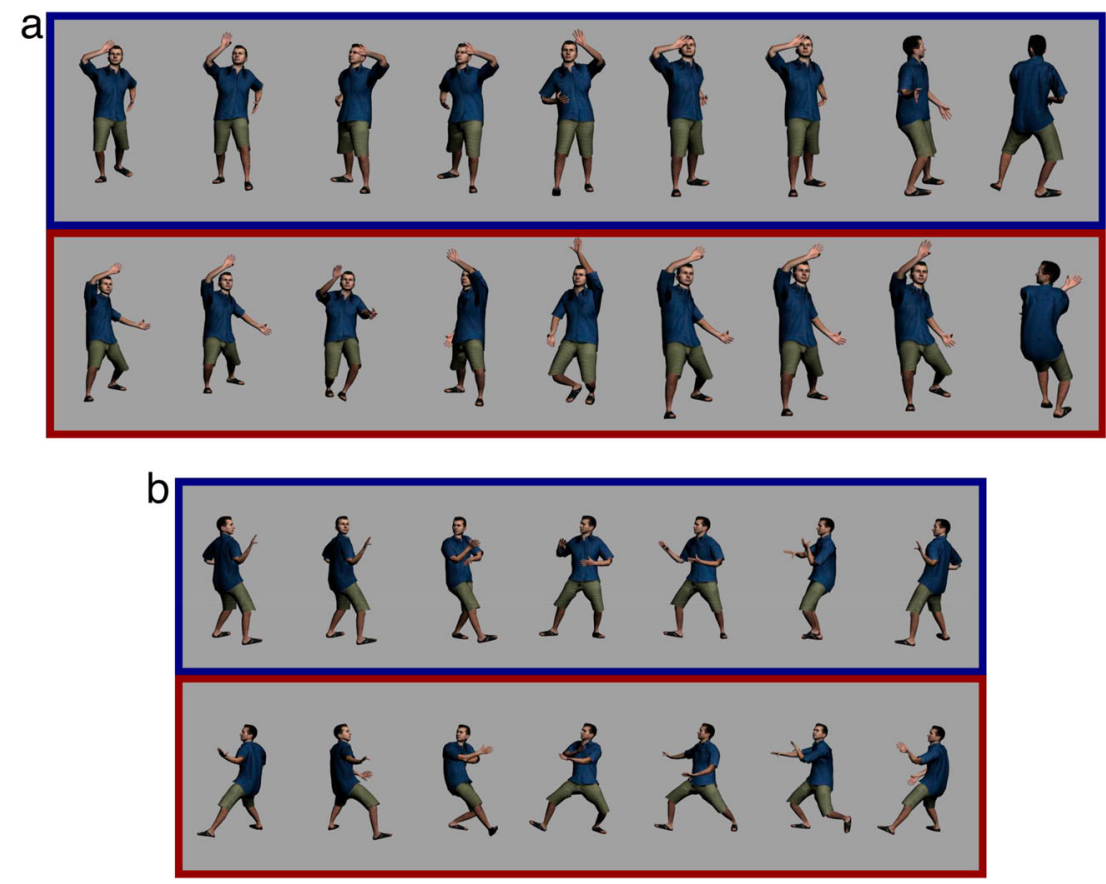

Figure 2. Test sequence 1 (a) and test sequence 2 (b) for both Denis' (red) and Joachim's (blue) motion.

asked to rate facial features and character traits but were not explicitly asked to judge the body or body motion. The learning stage took about 15 minutes, and after a short break, participants continued with the test stage.

During test, participants saw all 11 avatars, each animated with two sequences taken out of Denis' and Joachim's motion (Figure 2). The specific sequences were chosen, because they showed the face from frontal view. Sequence 1 consisted of 30 frames (frames 31-60 of the original sequence), resulting in a duration of 1.2 seconds; sequence 2 consisted of 50 frames (frames $401-450$ of the original sequence) resulting in a duration of 2 seconds. Each sequence was presented 16 times animated with each of the 11 heads, resulting in a total of 704 trials. Participants had to indicate as accurately as possible as to whether the avatar they saw looked more like Joachim or Denis by pressing J (for Joachim) or D (for Denis) on a standard German QWERTZ (as opposed to an English QWERTY) computer keyboard. The whole experiment took about 1.5 hours to complete.

\section{Results and analysis}

For each participant, logistic psychometric functions were fitted to the data for each condition (i.e., 2 sequences $\times 2$ body motions) using the Palamedes Toolbox (Prins \& Kingdom, 2009) for Matlab. Three participants were excluded as their psychometric functions had negative slopes, suggesting they were unable to perform the task as instructed. Analysis was thus performed on the data from the remaining 13 participants.

Figure 3 shows example psychometric functions for all four conditions from two participants (top) and the average psychometric functions for both sequences and body motions across all participants (bottom). Data are arbitrarily plotted as a function of Joachim responses. Two patterns seem immediately obvious. First, performance levels at the endpoints of the morph scale are always high (>80\%), suggesting that the training phase was successful in establishing the two facial identities. Second, participants were overall more likely to respond Joachim when the avatar was animated with Joachim's motion and more likely to respond Denis when the avatar was animated with Denis' motion, suggesting that body motion is also influencing performance.

We extracted values for the point of subjective equivalence (PSE) from each psychometric function. The PSE describes the perceptual midpoint of the morph sequence, i.e., the morph that is perceived as most ambiguous for each individual participant. The 

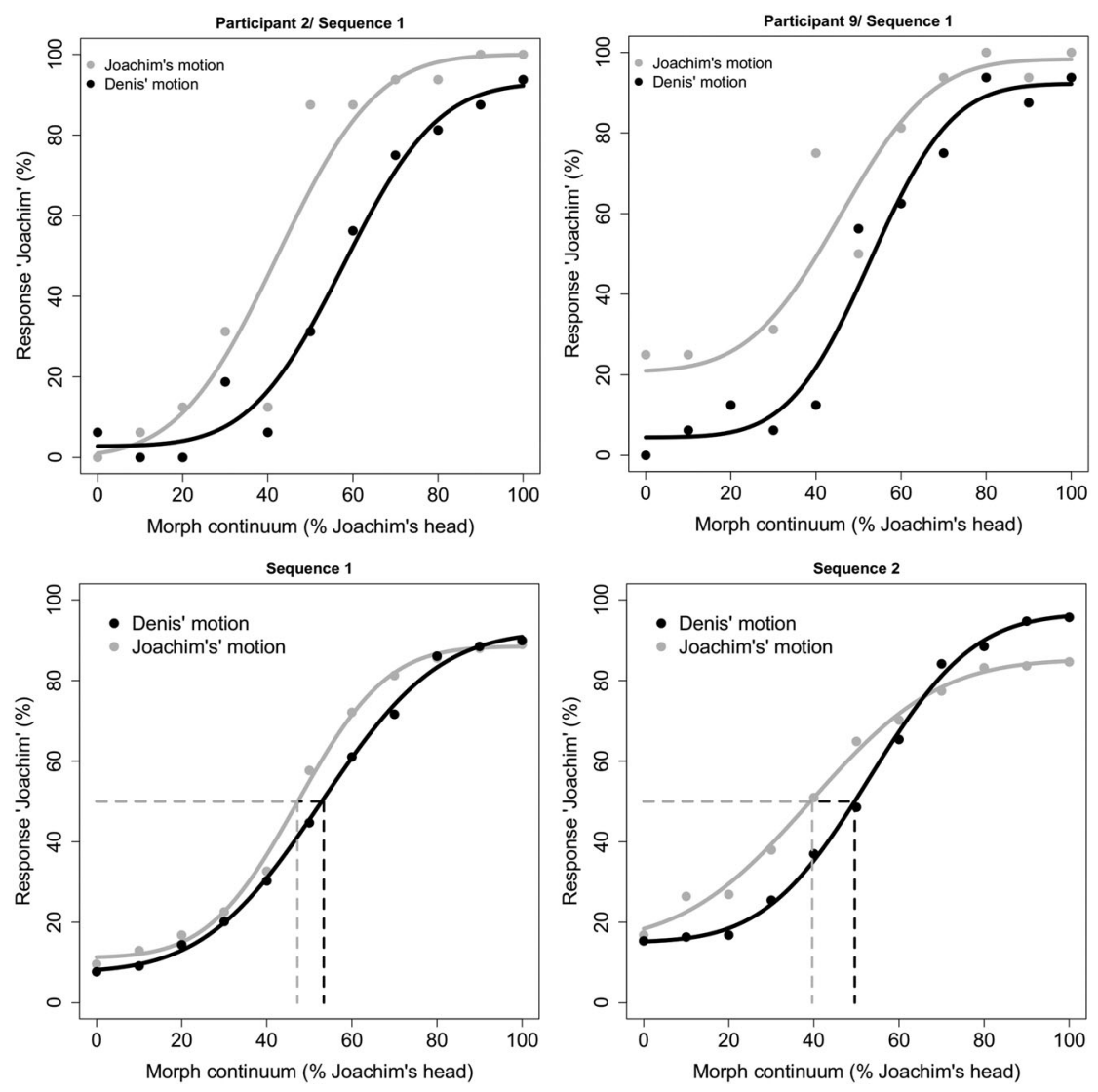

Figure 3. Example psychometric functions for participant 1 (top left) and participant 2 (top right) and average psychometric functions for sequence 1 (bottom left) and sequence 2 (bottom right). Dashed lines indicate PSEs for avatars animated with Denis' motion (black) and Joachim's motion (grey). The PSEs for avatars animated with Denis' motion are generally higher than those for avatars animated with Joachim's motion.

PSE does not necessarily coincide with the physically most ambiguous morph due to, for example, differences in the initial distinctiveness of the faces, or individual biases of the participants. A 2 (body motion) $\times 2$ (motion sequence) repeated measures ANOVA performed on the PSEs revealed a main effect of body motion $(F(1,12)=7.58$, $\left.p<.02 ; \eta^{2}=.387\right)$ but no effect of sequence $(F(1,12)=$ $\left.2.8, p=.12 ; \eta^{2}=.19\right)$, and no interaction $(F(1,12)=$ 2.03, $\left.p=.661 ; \eta^{2}=.01\right)$.

Figure 4 shows the average PSEs across all participants, measured in \% morph Joachim. The PSE for Denis' motion was overall higher $(M=50.87, S D=$ $10.57)$ than the PSE for Joachim's motion. $(M=40.6$, $S D=15.87)$. These results show that body motion affected identity decisions: the morph to which participants responded equally often with Joachim and Denis contained more facial identity from Joachim when it was animated with Denis' motion and more facial identity from Denis when it was animated with Joachim's motion.

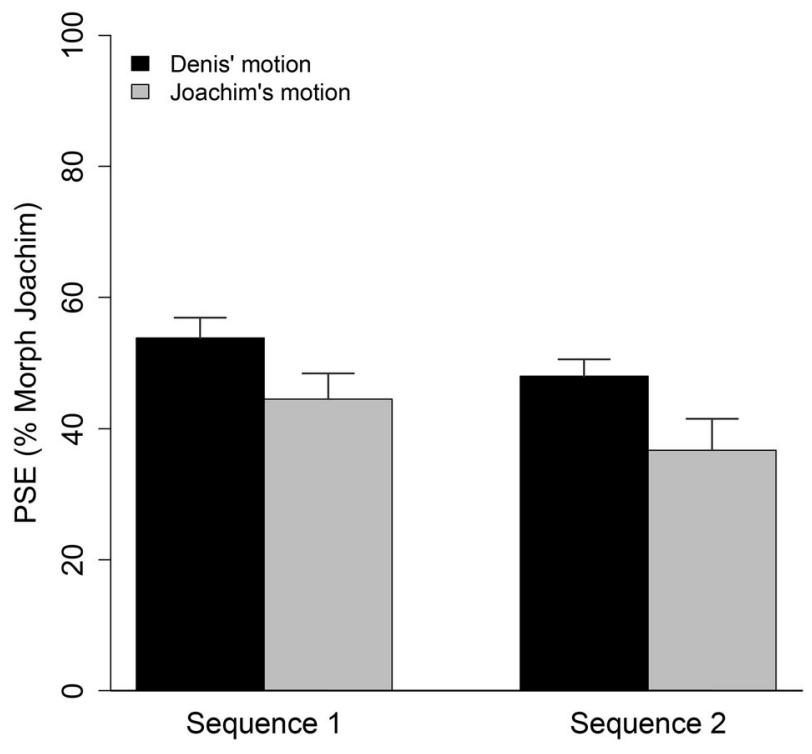

Figure 4. Mean PSE values for all participants for both motion sequences and body motions. Error bars represent standard errors from the mean. 


\section{Discussion}

In the current study we used animated whole body avatars to test the impact of individual motion on the recognition of identity. During a learning phase participants were familiarized with two individuals, Joachim and Denis. Their only discriminable features were their faces and unique body movements. The body itself, clothing and hairstyle were the same for all stimuli used in the experiment. In a subsequent recognition task, participants saw avatars whose facial identity consisted of morphs between the learned individuals. Across trials each avatar was seen animated with sequences taken from both of the learned movement patterns. Participants were asked to judge the identity of the avatars.

The avatars that contained the two original heads were predominantly identified by their facial identity regardless of body motion. More importantly however, when facial information was perceptually ambiguous, participants' identity decisions were predominantly based on the body motion. Interestingly, the PSEs were shifted away from the physical midpoint in that the perceptually ambiguous morphs overall contained more of Denis' identity. Such difference between the PSEs and the physical ambiguous morph could be due to a difference in distinctiveness between the two faces. Nevertheless, our results clearly show that body motion affects the perception of identity. These results are particularly interesting, because during learning, participants were not explicitly asked to attend to the body motion.

The current study makes two main contributions to the literature on person recognition. First, it introduces a new context within which to explore whole-person recognition. Using a combination of motion capture and computer animation techniques we have shown that it is possible to present full body stimuli in which one dimension (i.e., form or motion) can be held constant-rendering it uninformative-while varying the other. This powerful technique has previously been used to explore the influence of motion on facial identity decisions (Hill et al., 2003; Knappmeyer et al., 2003). Here, we have extended this work and demonstrate the technique's potential for studying the influence of body motion on wholeperson recognition.

Second, the current findings may shed light on the nature of the representations underlying form-motion integration. The question of how we integrate dynamic and static information has a long history, and has been particularly well studied within the contexts of object recognition (Chuang, Vuong, \& Bülthoff, 2012; Newell \& Findlay, 1997; Newell, Wallraven, \& Huber, 2004; Stone, 1998, 1999; Vuong, Friedman, \& Plante, 2009), biological motion (Giese \& Poggio, 2003; Mather, Pavan, Bellacosa Marotti, Campana, \& Casco, 2013; Troje, 2002) and face recognition (Butcher \& Lander, 2016; O'Toole, Roark, \& Abdi, 2002; Pilz et al., 2006; Thornton \& Kourtzi, 2002). Here, in extending these questions to the area of person recognition, we have demonstrated that facial-form can be integrated with body-motion during the computation of identity. To our knowledge, previous studies have only explored integration within the same identity components, that is facial-form to facial-motion and body-form to body-motion. The current findings thus suggest that integration mechanisms are likely to be very flexible, an idea we return to shortly.

It is, of course, important to acknowledge that the stimuli and task we have employed are a little unusual. Outside of the laboratory, we rarely encounter a range of heads being attached to a single body that can move with different motions! While this does raise questions about ecological validity, we argue that the reduction in certainty about facial identity introduced by morphing is likely to be very similar to that experienced when recognizing someone from a distance (Hahn et al., 2016; Yovel \& O'Toole, 2016) or in impoverished viewing conditions (Burton et al., 1999; Liu et al., 2003). Furthermore, using avatars is the only possibility to have a full, if uninformative, body structure (in contrast to point-light stimuli, for example) in addition to being able to precisely control graphics techniques (in contrast to video stimuli, for example).

In summary, our results clearly show that body motion affects the perception of identity, and support the hypothesis that body motion is encoded together with the identity of a person. Even though the face provides the main cue to identity, body motion seems to be especially employed when other important information is obscured, or less reliable. Our data support previous studies on person recognition that have suggested an integration of a variety of available identity cues such as, for example, facial, body, or movement information for 
an optimal encoding of identity (for review see Yovel \& O'Toole, 2016).

Having shown that body motion aids the encoding and later recognition of identity, the question remains as to how exactly it facilitates processing. For face recognition, two main mechanisms have been suggested to explain the effects of motion on identity processing (Knappmeyer et al., 2003; O'Toole et al., 2002; Roark, O'Toole, Abdi, \& Barrett, 2006). The representation enhancement hypothesis suggests that facial motion can aid recognition by enhancing the quality of the three-dimensional information available for a face (Christie \& Bruce, 1998; Pike, Kemp, Towell, \& Phillips, 1997). This hypothesis posits that motion does not contribute to a more robust representation per se but rather enhances the representation of the static structures of a face. This may be due to additional static information provided in the moving sequence. These mechanisms are directly perceptual and do not depend on prior experience with a face. Therefore, it applies equally well to personally familiar and unfamiliar faces.

While such a mechanism could be operating during the learning phase of the current study, it seems unlikely to account for the main pattern of results. That is, during familiarization, particular body postures and movement profiles could help to build more robust representations of each of the target identities by serving to expose the 3D structure of the faces. During testing however, such structural information is intentionally rendered ambiguous via morphing and each head is shown with sequences taken from both actors. While some mismatch in expectation about the structure of a face, driven by the body posture and movement could influence decisions, we suggest this would be a very subtle effect, almost certainly overridden by the mechanism we discuss next.

The supplemental information hypothesis suggests that characteristic motion patterns help us to identify faces because identity-specific facial motion is encoded together with the invariant structure of a face (Bruce \& Valentine, 1985; Christie \& Bruce, 1998; Hill et al., 2003; Knappmeyer et al., 2003; Lander \& Butcher, 2015; Lander, Christie, \& Bruce, 1999). Such idiosyncratic motion patterns help us to identify people we are well acquainted with. Non-rigid facial motion may provide characteristic cues to identityfor example, a particular way of smiling or indicating confusion-and/or may help to predict possible expressive configurations that a face may adopt. While this mechanism is mostly thought to aid the recognition of famous and personally familiar faces, several studies have shown that relatively brief familiarization with dynamic faces can establish characteristic movement effects (Knappmeyer et al., 2003; Maguinness \& Newell, 2014, 2015; Pilz et al., 2006)

The current study extends these findings and indicates that briefly familiarized characteristic body movements can be encoded along with invariant face and body cues to influence person recognition decisions. Previous studies with point-light figures (e.g., Cutting \& Kozlowski, 1977; Loula et al., 2005) and novel objects (e.g., Newell et al., 2004) have shown that motion can support identity decisions. Here, we have shown that this is so even in the presence of human form cues. A question that remains for future studies is to determine whether such motion and form information becomes fully integrated or simply remain as independent, but associated cues to identity. For example, an integrated account would suggest that our 50\% morph faces are seen as "looking like" one or other of the actors as a function of motion sequence. An independent account would argue that in situations where the face is uninformative, participants simply ignore this cue and base their decisions on the body motion.

While our current data do not allow us to distinguish between these two possibilities, elsewhere we have argued that form and motion are directly integrated into what Freyd (1987) termed "dynamic mental representations"-by explicitly taking into account both spatial and temporal dimensions, such representations are thought to more flexibly and robustly reflect real-world experience, giving rise to various forms of anticipatory and dynamic-over-static advantages (Knappmeyer et al., 2003; Pilz et al., 2006, 2011; Thornton \& Kourtzi, 2002). Pilz et al. (2011), for example, found that avatars animated with the identical looming motion-that is there were no characteristic movements-gave rise to facilitated recognition performance compared to static control conditions.

A dynamic representation of a person, to be specific, might be expected to encode not only the structural characteristics, but also the precise way the face and body can change over time, during facial expressions or body gestures, for example. The 
prevalence of dynamic events in our everyday experience can also be used to make an "evolutionary" argument concerning the nature of dynamic representations. The visual system is constantly exposed to faces and people in motion. Therefore, the neural mechanisms for encoding dynamic stimuli might be more robust than those for encoding evolutionaryrare static stimuli. Thus, relevant biological motion may help to build a dynamic representation of a person or face, which later on facilitates the recognition of that person across different viewing conditions and contexts.

Again, in the current experiment, we can only speculate that such representations are influencing behaviour, especially as our design did not directly contrast static and dynamic learning. It seems clear that characteristic motion aids the recognition of the ambiguous avatars, and we would argue that this is made possible due to the robust, dynamic encoding of the stimulus identity. Our suggestion, then is that some combination of these above mentioned mechanisms work in unison in that the motion of the stimuli generally leads to a robust encoding of the stimulus identity, whereas the characteristic body motion later aids the recognition of the ambiguous avatars.

Now that we have suggested how dynamic advantages may arise, we still need to clarify where and how such dynamic mental representations may be implemented.

One theory that could explain the emergence of dynamic representation is the common coding theory, which suggests that information from the visual and the motor system are integrated to form a single representation of, for example, specific actions (Prinz, 1997). The common coding theory mainly explains the planning and control of actions. However, it also provides a framework for action perception, and the involvement of the motor system when perceiving the actions of others might enhance the strength and reliability of dynamic representations. And indeed, it has been shown that motor and premotor areas are involved in observation of human movement (Grafton, Arbib, Fadiga, \& Rizzolatti, 1996; Rizzolatti et al., 1996), even in the form of point-light stimuli (Saygin, Wilson, Hagler, Bates, \& Sereno, 2004).

Another theory that could help explain the emergence of dynamic mental representations is Giese and Poggio's model on the processing of biological motion (Giese \& Poggio, 2003). Giese and Poggio provide an explanation for the processing of biological motion on the basis of the dorsal and the ventral visual pathways (Creem \& Proffitt, 2001; Ungerleider \& Mishkin, 1982). The ventral pathway projects from the primary visual cortex (V1) to the inferotemporal cortex and is mainly thought to be involved in the processing of static visual input, i.e., information about form. The dorsal pathway projects from the primary visual areas into the posterior parietal cortex and is involved in the processing of dynamic visual input, e.g., optic flow information. Although these streams are largely interconnected, the extent of their interaction is unknown. Giese and Poggio (2003) argue that both pathways are involved in the processing of biological motion (also see Lange \& Lappe, 2006). The dorsal motion pathway recognizes biological motion by analysing optic-flow pattern, whereas the ventral form pathway analyses biological motion by recognizing sequences of snapshots of body shapes. The analysis is based on processing increasingly more complex features in both pathways. Amongst evidence from various psychophysical and brain imaging studies on biological motion (Bertenthal \& Pinto, 1994; Grossman \& Blake, 2002; Grossman et al., 2000; Kozlowski \& Cutting, 1977), the hypothesis is underlined by patient studies that showed that patients with lesions in the dorsal pathways are still able to recognize point-light stimuli (McLeod, 1996; Vaina, Lemay, Bienfang, Choi, \& Nakayama, 1990). These results strongly suggest that the form pathway is also involved in the processing of motion information. The superior temporal sulcus (STS) has long been suggested as a potential location of integrating information from both pathways, and has been shown to respond to body and facial form and motion information (Giese \& Poggio, 2003; Grossman et al., 2000; Oram \& Perrett, 1996; Schultz \& Pilz, 2009; Yovel \& O'Toole, 2016).

The integration of form and motion information could explain the origin of previously reported dynamic advantages for facial and body motion. The mental representation of a behaviourally relevant and biologically plausible dynamic stimulus is potentially more robust, because the stimulus is processed by both pathways simultaneously, and both form and motion information are integrated into a single representation. Recent studies have shown that with 
regards to faces both form and motion pathway are involved in processing facial motion (Pitcher, Dilks, Saxe, Triantafyllou, \& Kanwisher, 2011; Schultz \& Pilz, 2009; Schultz, Brockhaus, Bülthoff, \& Pilz, 2013). Whereas activation in the form pathway seems to be more related to processing the amount of information available in the motion sequence the motion pathway seems to be predominantly involved in processing the change over time, i.e., activation in the form pathway is related to the number of frames presented, whereas activation in the motion pathway is related to the consistency in change over time. Such mechanisms might similarly relate to body motion as used in our study. However, future studies will have to investigate this in further detail.

\section{Conclusions}

The current results provide evidence for the importance of body motion for the encoding and later recognition of identity. In addition, our results provide evidence that it is important to move away from only investigating the impact of facial information for person recognition and demonstrate that it is important to take into account the face in the context of a body. Current advances in animation techniques and virtual reality make it possible to take apart different sources of information and to investigate their differential impact on, for example, facial and body motion information. It seems likely that such virtual reality (VR) animation tools will become more standard in psychological experiments as they allow close approximations to real world scenarios with the possibility to fully control experimental conditions

\section{Acknowledgements}

We would like to thank Martin Giese for providing the motion capture data.

\section{Disclosure statement}

No potential conflict of interest was reported by the authors.

\section{References}

Agnew, H. C., Phillips, L. H., \& Pilz, K. S. (2016). Global form and motion processing in healthy ageing. Acta Psychologica, 166, 12-20. doi:10.1016/j.actpsy.2016.03.005
Bertenthal, B. I., \& Pinto, J. (1994). Global processing of biological motions. Psychological Science, 5(4), 221-224. doi:10. 1111/j.1467-9280.1994.tb00504.x

Blake, R., \& Shiffrar, M. (2007). Perception of human motion. Annual Review of Psychology, 58, 47-73. doi:10.1146/ annurev.psych.57.102904.190152

Blanz, V., \& Vetter, T. (1999). A morphable model for the synthesis of 3D faces. In Proceedings of the 26th annual conference on computer graphics and interactive techniques Siggraph "99 (pp. 187-194). New York, NY: ACM Press. doi:10.1145/311535.311556

Bower, G. H., \& Karlin, M. B. (1974). Depth of processing pictures of faces and recognition memory. Journal of Experimental Psychology, 103(4), 751-757. doi:10.1037/h0037190

Bruce, V., Henderson, Z., Newman, C., \& Burton, A. M. (2001). Matching identities of familiar and unfamiliar faces caught on CCTV images. Journal of Experimental Psychology: Applied, 7(3), 207-218. Retrieved from http://www.ncbi.nlm. nih.gov/pubmed/11676099

Bruce, V., \& Valentine, T. (1985). Identity priming in the recognition of familiar faces. British Journal of Psychology (London, England: 1953), 76(Pt 3), 373-383. Retrieved from http://www.ncbi.nlm.nih.gov/pubmed/4041705

Bruce, V., \& Young, A. W. (2012). Face perception. East Sussex: Psychology Press.

Burton, A. M., Wilson, S., Cowan, M., \& Bruce, V. (1999). Face recognition in poor-quality video: Evidence from security surveillance. Psychological Science, 10(3), 243-248. doi:10.1111/ 1467-9280.00144

Butcher, N., \& Lander, K. (2016). Exploring the motion advantage: Evaluating the contribution of familiarity and differences in facial motion. The Quarterly Journal of Experimental Psychology, 1-11. doi:10.1080/17470218.2016.1138974

Christie, F., \& Bruce, V. (1998). The role of dynamic information in the recognition of unfamiliar faces. Memory \& Cognition, 26 (4), 780-790. Retrieved from http://www.ncbi.nlm.nih.gov/ pubmed/9701969

Chuang, L. L., Vuong, Q. C., \& Bülthoff, H. H. (2012). Learned Nonrigid object motion is a view-invariant cue to recognizing novel objects. Frontiers in Computational Neuroscience, 6, 26. doi:10.3389/fncom.2012.00026

Creem, S. H., \& Proffitt, D. R. (2001). Defining the cortical visual systems: "What", "where", and "how". Acta Psychologica, 107 (1-3), 43-68. Retrieved from http://www.ncbi.nlm.nih.gov/ pubmed/11388142

Cutting, J. E., \& Kozlowski, L. T. (1977). Recognizing friends by their walk: Gait perception without familiarity cues. Bulletin of the Psychonomic Society, 9(5), 353-356. doi:10.3758/ BF03337021

Dittrich, W. H., Troscianko, T., Lea, S. E. G., \& Morgan, D. (1996). Perception of emotion from dynamic point-light displays represented in dance. Perception, 25(6), 727-738. doi:10. 1068/p250727

Freyd, J. J. (1987) Dynamic mental representations. Psychological Review, 94, 427-438. doi:10.1037/0033-295X.94.4.427

Gesellensetter, L., Krämer, N. C., \& Wachsmuth, I. (2005). A conversational agent as museum guide - Design and evaluation 
of a real-world application. The 5th International Working Conference on Intelligent Virtual Agents. Retrieved from http://citeseerx.ist.psu.edu/viewdoc/summary?doi=10.1.1. 93.7488

Giese, M. A., \& Poggio, T. (2003). Cognitive neuroscience: Neural mechanisms for the recognition of biological movements. Nature Reviews: Neuroscience, 4(3), 179-192. doi:10.1038/ nrn 1057

Grafton, S. T., Arbib, M. A., Fadiga, L., \& Rizzolatti, G. (1996). Localization of grasp representations in humans by positron emission tomography 2. Observation compared with imagination. Experimental Brain Research, 112(1), 103-111. Retrieved from http://www.ncbi.nlm.nih.gov/pubmed/8951412

Griesser, R. T., Cunningham, D. W., Wallraven, C., \& Bülthoff, H. H. (2007). Psychophysical investigation of facial expressions using computer animated faces. In Proceedings of the 4th symposium on applied perception in graphics and visualization - APGV “07 (p. 11). New York, NY: ACM Press. doi:10.1145/ 1272582.1272585

Grossman, E. D., \& Blake, R. (2002). Brain areas active during visual perception of biological motion. Neuron, 35(6), 11671175. Retrieved from http://www.ncbi.nlm.nih.gov/pubmed/ 12354405

Grossman, E. D., Donnelly, M., Price, R., Pickens, D., Morgan, V., Neighbor, G., \& Blake, R. (2000). Brain areas involved in perception of biological motion. Journal of Cognitive Neuroscience, 12(5), 711-720. Retrieved from http://www. ncbi.nlm.nih.gov/pubmed/11054914

Hahn, C. A., O'Toole, A. J., \& Phillips, P. J. (2016). Dissecting the time course of person recognition in natural viewing environments. British Journal of Psychology (London, England: 1953), 107(1), 117-134. doi:10.1111/bjop.12125

Hill, H., Jinno, Y., \& Johnston, A. (2003). Comparing solid-body with point-light animations. Perception, 32(5), 561-566. Retrieved from http://www.ncbi.nlm.nih.gov/pubmed/12854642

Johansson, G. (1973). Visual perception of biological motion and a model for its analysis. Perception \& Psychophysics, 14 (2), 201-211. doi:10.3758/BF03212378

Johnson, K. L., \& Shiffrar, M. (2013). People watching: Social perceptual, and neurophysiological studies of body perception. New York, NY: Oxford University Press.

Kao, D., \& Harrell, D. F. (2015). Exploring the impact of role model avatars on game experience in educational games. In Proceedings of the 2015 annual symposium on computerhuman interaction in play - CHI PLAY "15 (pp. 571-576). New York, NY: ACM Press. doi:10.1145/2793107.2810291

Knappmeyer, B., Thornton, I. M., \& Bülthoff, H. H. (2003). The use of facial motion and facial form during the processing of identity. Vision Research, 43(18), 1921-1936. Retrieved from http://www.ncbi.nlm.nih.gov/pubmed/12831755

Knoblich, G., Thornton, I. M., Grosjean, F., \& Shiffrar, M. (2006). Human body perception from the inside Out. USA: OUP. Retrieved from https://books.google.com/books?hl=en\&lr= \&id=iRkCDYXRRXOC\&pgis $=1$

Kozlowski, L. T., \& Cutting, J. E. (1977). Recognizing the sex of a walker from a dynamic point-light display. Perception \& Psychophysics, 21(6), 575-580. doi:10.3758/BF03198740
Lander, K., \& Butcher, N. (2015). Independence of face identity and expression processing: Exploring the role of motion. Frontiers in Psychology, 6, 255. doi:10.3389/fpsyg.2015.00255

Lander, K., Christie, F., \& Bruce, V. (1999). The role of movement in the recognition of famous faces. Memory \& Cognition, 27 (6), 974-985. Retrieved from http://www.ncbi.nlm.nih.gov/ pubmed/10586574

Lange, J., \& Lappe, M. (2006). A model of biological motion perception from configural form cues. The Journal of Neuroscience: The Official Journal of the Society for Neuroscience, 26(11), 2894-2906. doi:10.1523/JNEUROSCI. 4915-05.2006

Liu, C. H., Seetzen, H., Burton, A. M., \& Chaudhuri, A. (2003). Face recognition is robust with incongruent image resolution: Relationship to security video images. Journal of Experimental Psychology: Applied, 9(1), 33-41. Retrieved from http://www.ncbi.nlm.nih.gov/pubmed/12710836

Loula, F., Prasad, S., Harber, K., \& Shiffrar, M. (2005). Recognizing people from their movement. Journal of Experimental Psychology: Human Perception and Performance, 31(1), 210220. doi:10.1037/0096-1523.31.1.210

Maguinness, C., \& Newell, F. N. (2014). Motion facilitates face perception across changes in viewpoint and expression in older adults. Journal of Experimental Psychology: Human Perception and Performance, 40(6), 2266-2280. doi:10.1037/ a0038124

Maguinness, C., \& Newell, F. N. (2015). Non-rigid, but not rigid, motion interferes with the processing of structural face information in developmental prosopagnosia. Neuropsychologia 70, 281-295. doi:10.1016/j.neuropsychologia.2015.02.038

Mather, G., Pavan, A., Bellacosa Marotti, R., Campana, G., \& Casco, C. (2013). Interactions between motion and form processing in the human visual system. Frontiers in Computational Neuroscience, 7, 65. doi:10.3389/fncom.2013. 00065

McLeod, P. (1996). Preserved and impaired detection of structure from motion by a "Motion-blind" patient. Visual Cognition, 3(4), 363-392. doi:10.1080/135062896395634

Newell, F. N., \& Findlay, J. M. (1997). The effect of depth rotation on object identification. Perception, 26(10), 1231-1257. Retrieved from http://www.ncbi.nlm.nih.gov/pubmed/ 9604061

Newell, F. N., Wallraven, C., \& Huber, S. (2004). The role of characteristic motion in object categorization. Journal of Vision, 4(2), 118-129. doi:10.1167/4.2.5

Oram, M. W., \& Perrett, D. I. (1996). Integration of form and motion in the anterior superior temporal Polysensory area (STPa) of the macaque monkey. Journal of Neurophysiology, 76(1), 109-129. Retrieved from http://www.ncbi.nlm.nih. gov/pubmed/8836213

O'Toole, A. J., Roark, D. A., \& Abdi, H. (2002). Recognizing moving faces: a psychological and neural synthesis. Trends in Cognitive Sciences, 6(6), 261-266. Retrieved from http:// www.ncbi.nlm.nih.gov/pubmed/12039608

Panayiotopoulos, T., Gratch, J., Aylett, R., Ballin, D., Olivier, P., \& Rist, T. (Eds.). (2005). Intelligent virtual agents (Vol. 3661). Berlin: Springer Berlin Heidelberg. doi:10.1007/11550617 
Pike, G. E., Kemp, R. I., Towell, N. A., \& Phillips, K. C. (1997). Recognizing moving faces: The relative contribution of motion and perspective view information. Visual Cognition, 4, 409-437. doi:10.1080/713756769

Pilz, K. S., Bülthoff, H. H., \& Vuong, Q. C. (2009). Learning influences the encoding of static and dynamic faces and their recognition across different spatial frequencies. Visual Cognition, 17(5), 716-735. doi:10.1080/13506280802340588

Pilz, K. S., Thornton, I. M., \& Bülthoff, H. H. (2006). A search advantage for faces learned in motion. Experimental Brain Research, 171(4), 436-447. doi:10.1007/s00221-005-0283-8

Pilz, K. S., Vuong, Q. C., Bülthoff, H. H., \& Thornton, I. M. (2011). Walk this way: Approaching bodies can influence the processing of faces. Cognition, 118(1), 17-31. doi:10.1016/j. cognition.2010.09.004

Pitcher, D., Dilks, D. D., Saxe, R. R., Triantafyllou, C., \& Kanwisher, N. (2011). Differential selectivity for dynamic versus static information in face-selective cortical regions. Neurolmage, 56(4), 2356-2363. doi:10.1016/j.neuroimage. 2011.03.067

Pollick, F. E., Kay, J. W., Heim, K., \& Stringer, R. (2005). Gender recognition from point-light walkers. Journal of Experimental Psychology: Human Perception and Performance, 31(6), 1247-1265. doi:10.1037/0096-1523.31.6.1247

Powell, W. A., Corbett, N., \& Powell, V. (2016). Creative technologies for multidisciplinary applications. A. M. Connor \& S. Marks (Eds.). IGI Global. doi:10.4018/978-1-5225-0016-2

Prins, N., \& Kingdom, F. A. A. (2009). Palamedes: Matlab routines for analyzing psychophysical data.

Prinz, W. (1997). Perception and action planning. European Journal of Cognitive Psychology, 9, 129-154.

Rice, A., Phillips, P. J., \& O'Toole, A. (2013). The role of the face and body in unfamiliar person identification. Applied Cognitive Psychology, 27(6), 761-768. doi:10.1002/acp.2969

Rizzolatti, G., Fadiga, L., Matelli, M., Bettinardi, V., Paulesu, E., Perani, D., \& Fazio, F. (1996). Localization of grasp representations in humans by PET: 1. Observation versus execution. Experimental Brain Research, 111(2), 246-252. doi:10.1007/ BF00227301

Roark, D. A., O'Toole, A. J., Abdi, H., \& Barrett, S. E. (2006). Learning the moves: The effect of familiarity and facial motion on person recognition across large changes in viewing format. Perception, 35(6), 761-773. Retrieved from http://www.ncbi.nlm.nih.gov/pubmed/16836043

Robbins, R. A., \& Coltheart, M. (2015). The relative importance of heads, bodies, and movement to person recognition across development. Journal of Experimental Child Psychology, 138, 1-14. doi:10.1016/j.jecp.2015.04.006

Saygin, A. P., Wilson, S. M., Hagler, D. J., Bates, E., \& Sereno, M. I. (2004). Point-light biological motion perception activates human premotor cortex. The Journal of Neuroscience: The Official Journal of the Society for Neuroscience, 24(27), 61816188. doi:10.1523/JNEUROSCI.0504-04.2004

Schultz, J., Brockhaus, M., Bülthoff, H. H., \& Pilz, K. S. (2013). What the human brain likes about facial motion. Cerebral Cortex (New York, N.Y.: 1991), 23(5), 1167-1178. doi:10.1093/ cercor/bhs106
Schultz, J., \& Pilz, K. S. (2009). Natural facial motion enhances cortical responses to faces. Experimental Brain Research, 194 (3), 465-475. doi:10.1007/s00221-009-1721-9

Simhi, N., \& Yovel, G. (2016). The contribution of the body and motion to whole person recognition. Vision Research, 122, 12-20. doi:10.1016/j.visres.2016.02.003

Spanlang, B., Navarro, X., Normand, J.-M., Kishore, S., Pizarro, R., \& Slater, M. (2013). Real time whole body motion mapping for avatars and robots. In Proceedings of the 19th ACM symposium on virtual reality software and technology - VRST "13 (p. 175). New York, NY: ACM Press. doi:10.1145/2503713.2503747

Spencer, J. M. Y., Sekuler, A. B., Bennett, P. J., Giese, M. A., \& Pilz, K. S. (2016). Effects of aging on identifying emotions conveyed by point-light walkers. Psychology and Aging. doi:10.1037/a0040009

Stone, J. V. (1998). Object recognition using spatiotemporal signatures. Vision Research, 38(7), 947-951. Retrieved from http://www.ncbi.nlm.nih.gov/pubmed/9666977

Stone, J.V. (1999). Object recognition: View-specificity and motionspecificity. Vision Research, 39(24), 4032-4044. Retrieved from http://www.ncbi.nlm.nih.gov/pubmed/10748936

Thompson, J., \& Parasuraman, R. (2012). Attention, biological motion, and action recognition. Neurolmage, 59(1), 4-13. doi:10.1016/j.neuroimage.2011.05.044

Thornton, I. M. (2006). Biological motion: Point-light walkers and beyond. In G. Knoblich, I. M. Thornton, F. Grosjean, \& M. Shiffrar (Eds.), Human body perception from the inside out (pp. 271-303). Oxford: Oxford University Press.

Thornton, I. M., \& Kourtzi, Z. (2002). A matching advantage for dynamic human faces. Perception, 31(1), 113-132. Retrieved from http://www.ncbi.nlm.nih.gov/pubmed/11922118

Troje, N. F. (2002). Decomposing biological motion: A framework for analysis and synthesis of human gait patterns. Journal of Vision, 2(5), 371-387. doi:10.1167/2.5.2

Ungerleider, L. G., \& Mishkin, M. (1982). Two cortical visual systems. D. J. Ingle, M. A. Goodale, \& R. J. W. Mansfield (Eds.) (Analysis o.). MIT Press. Retrieved from http://www. oalib.com/references/14583922

Vaina, L. M., Lemay, M., Bienfang, D. C., Choi, A. Y., \& Nakayama, K. (1990). Intact "biological motion" and "structure from motion" perception in a patient with impaired motion mechanisms: A case study. Visual neuroscience, 5(04), 353-369. doi:10.1017/S0952523800000444

Vanrie, J., \& Verfaillie, K. (2004). Perception of biological motion: A stimulus set of human point-light actions. Behavior Research Methods, Instruments, \& Computers, 36(4), 625629. doi:10.3758/BF03206542

Vuong, Q. C., Friedman, A., \& Plante, C. (2009). Modulation of viewpoint effects in object recognition by shape and motion cues. Perception, 38(11), 1628-1648. Retrieved from http://www.ncbi.nlm.nih.gov/pubmed/20120262

Wallraven, C., Breidt, M., Cunningham, D. W., \& Bülthoff, H. H. (2008). Evaluating the perceptual realism of animated facial expressions. ACM Transactions on Applied Perception, 4(4), 1-20. doi:10.1145/1278760.1278764

Yovel, G., \& O'Toole, A. J. (2016). Recognizing people in motion. Trends in Cognitive Sciences, 20(5), 383-395. doi:10.1016/j.tics. 2016.02.005 